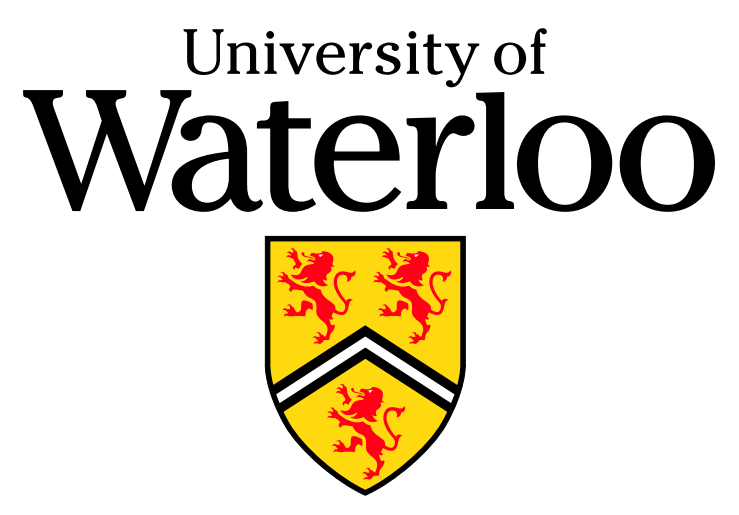

\title{
Sum-Rate Maximization in Single-Hop Wireless Networks with the On-Off Power Scheme
}

\author{
by \\ Jamshid Abouei, Alireza Bayesteh, Masoud Ebrahimi and Amir K. Khandani \\ Electrical and Computer Engineering Department \\ University of Waterloo \\ Waterloo, Ontario, Canada \\ Email: \{jabouei, alireza, masoud, khandani\}@cst.uwaterloo.ca
}

Technical Report UW-ECE \#2007-14

April 2007

(C)Jamshid Abouei, Alireza Bayesteh, Masoud Ebrahimi and A. K. Khandani 2007 

Library and Archives
Canada
Bibliothèque et Archives Canada

\section{RECEIPT FOR LEGAL DEPOSIT REÇU POUR DÉPÔT LÉGAL}

Legal deposit number

Numéro de dépôt léga

D

960741

al.] Khandani, Amir

Quantity received:

Quantité reçue:
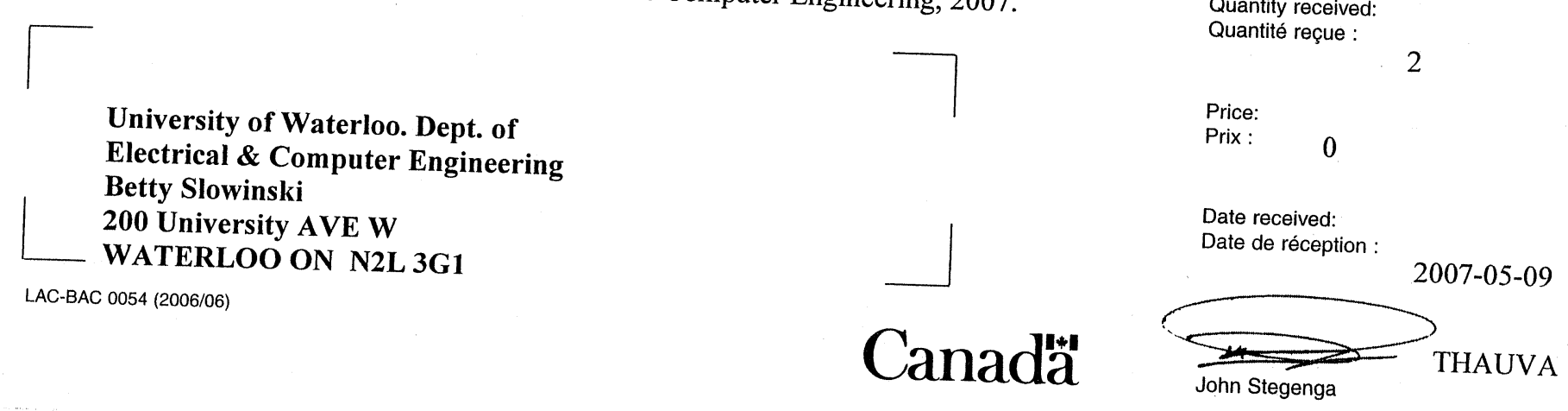

$G(0 \square$ 


\title{
Sum-Rate Maximization in Single-Hop Wireless Networks with the On-Off Power Scheme
}

\author{
Jamshid Abouei, Alireza Bayesteh, Masoud Ebrahimi, and Amir K. \\ Khandani
}

\begin{abstract}
A single-hop wireless network with $K$ links is considered, where the links are partitioned into $M$ clusters, each operating in a subchannel with bandwidth $\frac{W}{M}$. We assume that the links in each cluster perform the on-off power allocation strategy proposed in [1]. The problem is to analyze the average sum-rate of the network in terms of $M$ and under the shadow-fading effect with probability $\alpha$. It is demonstrated that for $M \sim o(K)$ and $0<\alpha \leq 1$, where $\alpha$ is fixed, the average sum-rate of the network scales as $\frac{W}{\alpha} \log \frac{K}{M}$. For $M \sim \Theta(K)$, we present an upper bound for the network throughput. It is proved that the maximum average sum-rate of the network for every value of $1 \leq M \leq K$ and $0<\alpha \leq 1$ is achieved at $M=1$. In fact, in the proposed model, partitioning the bandwidth $W$ into $M$ subchannels has no gain in terms of enhancing the throughput.
\end{abstract}

Financial support provided by Nortel and the corresponding matching funds by the Natural Sciences and Engineering Research Council of Canada (NSERC), and Ontario Centres of Excellence (OCE) are gratefully acknowledged.

The authors are affiliated with the Coding and Signal Transmission Laboratory, Electrical and Computer Engineering Department, University of Waterloo, Waterloo, ON, N2L 3G1, Canada, Tel: 519-884-8552, Fax: 519-888-4338, Emails: \{jabouei, alireza, masoud, khandani\}@cst.uwaterloo.ca. 


\section{Index Terms}

Sum-rate maximization, on-off power scheme, shadow-fading, wireless networks.

\section{INTRODUCTION}

The main challenge in multi-user wireless networks originates from the sharing of a common transmission bandwidth by users such that the network throughput is maximized. Since the throughput of the network is limited by the interference, several techniques are proposed to mitigate the interference. Effective spectral usage and power control in transmitters have long been regarded as efficient tools to reduce the interference and improve the throughput of the network with limited bandwidth. In recent years, various power control and spectrum sharing schemes have been extensively studied in cellular and multihop wireless networks [2]-[6]. For example in [3], the authors provide a comprehensive survey in the area of resource allocation, in particular in the context of fixed, dynamic and hybrid channel assignments for various wireless networks. Much of these works rely on centralized and cooperative algorithms. Due to significant challenges in using the centralized approaches, the attention of the researchers have drawn to the distributed resource allocation schemes [7]-[11]. Etkin and Tse [8] develop power and spectrum allocation strategies in multiple wireless systems. Under the assumptions of the omniscient nodes and strong interference, they show that frequency division multiplexing (FDM) is the optimal scheme in the sense of the throughput maximization.

In this paper, we study the performance of a single-hop wireless network, in which $K$ links are partitioned into $M$ clusters, each operating in a subchannel with bandwidth $\frac{W}{M}$. The users utilize the decentralized on-off power allocation scheme proposed in [1] and [7]. In [1] and [7], the authors study the performance of the network only for $M=1$ and under a Rayleigh-fading channel model. It is wellknown, however, that the wireless channel can be modeled in a more realistic manner. Here, we consider the shadow-fading effect that are caused by obstacles. Shadowing effect occurs when a node moves between obstructions and experiences fluctuations 
in its signal power. The main contribution of this work is to determine the maximum throughput of the network in terms of different values of $M$ and the probability of the shadowing effect, $\alpha$. Our strategy differs from the model studied in [9] and [12]; primarily we use a decentralized on-off power allocation scheme for a single-hop wireless network with $M$ subchannels, while [9] and [12] present a model with random connections for $M=1$ and using relay nodes.

Under the assumption of Rayleigh fading, an asymptotic analysis is carried out to show that for $M \sim o(K)$ and $0<\alpha \leq 1$, where $\alpha$ is a fixed value, the average sumrate of the network is of order $\frac{W}{\alpha} \log \frac{K}{M}$. It is shown that the maximum throughput of the network for every value of $M$ and $0<\alpha \leq 1$ is achieved at $M=1$.

The rest of the paper is organized as follows. In Section II, the network model and objectives are described. We analyze the average sum-rate of the network in terms of $M$ and $\alpha$ in Section III. Finally, in Section IV, an overview of the results and conclusions is presented, and directions for ongoing and future research are mentioned.

Knuth's notation [13]: For any functions $f(n)$ and $g(n)$ :

- $f(n)=O(g(n))$ means that $\lim _{n \rightarrow \infty}|f(n) / g(n)|<\infty$.

- $f(n)=\omega(g(n))$ means that $\lim _{n \rightarrow \infty}|f(n) / g(n)|=\infty$.

- $f(n)=o(g(n))$ means that $\lim _{n \rightarrow \infty}|f(n) / g(n)|=0$.

- $f(n)=\Theta(g(n))$ means that $\lim _{n \rightarrow \infty}|f(n) / g(n)|=c$, where $0<c<\infty$.

Also, $\log ($.$) is the natural logarithm function and \mathcal{N}=\{1,2, \ldots, n\}$.

\section{Network Model And ObJectives}

In this work, we consider a single-hop wireless network consisting of $K$ pairs of nodes $^{1}$, operating in a bandwidth $W$. The links are assumed to be partitioned into $M$ clusters such that the number of links in each cluster is the same. Also, the links are randomly divided among the clusters. The bandwidth $W$ is divided into $M$ disjoint subchannels, each with bandwidth $\frac{W}{M}$. Letting $\mathcal{C}_{j}$ denote cluster $j$, the links in $\mathcal{C}_{j}$

\footnotetext{
${ }^{1}$ The term "pair" is used to describe the transmitter and the related receiver, and "user" only for the transmitter.
} 
operate in subchannel $j$. In this work, we assume that $M$ is a variable parameter in the range of 1 to $K$. We also assume the number of links in each cluster, $n=\frac{K}{M}$, is a known information for the users. The link between transmitter $k$ and receiver $i$ in $\mathcal{C}_{j}$ is represented by the random variable $\mathcal{L}_{k i}^{(j)}$. Under a Rayleigh fading channel model, $\mathcal{L}_{k i}^{(j)}=h_{i i}^{(j)}$, for $k=i$. Also for $k \neq i$, the cross-channel gains are defined based on the shadow-fading model as follows ${ }^{2}$

$$
\mathcal{L}_{k i}^{(j)}= \begin{cases}h_{k i}^{(j)}, & \text { with probability } \alpha \\ 0, & \text { with probability } 1-\alpha .\end{cases}
$$

We consider a quasi-static block fading channel model, where the channel strength denoted by $h_{k i}^{(j)}=\left|g_{k i}^{(j)}\right|^{2}$ remains constant while transmitting one block and changes independently from block to block. Under a Rayleigh fading channel, $h_{k i}^{(j)}$ 's are exponentially distributed with unit mean. We also assume that the channel is flat fading. In other words, all the channels are assumed to be constant over the whole bandwidth $W$. We also assume that each receiver knows only its direct channel gain. This channelstate information (CSI) is fed back to the corresponding transmitter without any error.

In this work, we assume that all the links utilize the on-off power allocation strategy. Based on this scheme, the average transmit power of user $i$ is assumed to be $p_{i} \in\{0,1\}$. The power of additive white Gaussian noise (AWGN) at each receiver is assumed to be $\frac{N_{0} W}{M}$. Since the maximum transmit power is one, $\frac{N_{0} W}{M}$ is equivalent to $\frac{1}{S N R}$, where SNR is the signal to noise ratio. Assuming Gaussian signal transmission, the interference term will be Gaussian with power

$$
I_{i}^{(j)}=\sum_{\substack{k \neq i \\ k=1}}^{n} \mathcal{L}_{k i}^{(j)} p_{k}, \quad i, k \in \mathcal{C}_{j} .
$$

Due to the orthogonality of the allocated subchannels, no interference is imposed from links in $\mathcal{C}_{k}$ on links in $\mathcal{C}_{j}, k \neq j$. Under these assumptions, the achievable data

\footnotetext{
${ }^{2}$ It is worth to mention that the superscript $j$ means that the channel gains belong to cluster $j$, and it does not mean that the fading channel model is frequency-selective.
} 
rate of each link is expressed as

$$
R_{i}^{(j)}=\frac{W}{M} \log \left(1+\frac{h_{i i}^{(j)} p_{i}}{I_{i}^{(j)}+\frac{N_{0} W}{M}}\right), \quad i \in \mathcal{N} .
$$

We consider a homogeneous network in the sense that all the links have the same configurations and use the same protocols. Thus, the transmission strategy for all the nodes are agreed in advance. Next, we summarize the on-off power allocation strategy in a single-hop wireless network proposed in [1] and [7].

On-Off Power Allocation Strategy: In each cluster, all users perform the following steps during each block:

1- Based on the direct channel gain, the transmission policy is

$$
p_{i}= \begin{cases}1, & \text { if } h_{i i}^{(j)}>\tau_{n} \\ 0, & \text { Otherwise }\end{cases}
$$

where $\tau_{n}$ is a prespecified threshold level.

2- After adjusting the powers, each active user in $\mathcal{C}_{j}$ transmits a pilot signal with full power. All the receivers measure the interference and compute the rate using (2). Then, each receiver feedbacks the rate to its corresponding transmitter.

3- The active user transmits data with the computed rate and with full power.

In order to analyze the performance of the network, we define the network throughput as the average sum-rate. Letting $\bar{R}_{\text {sum }}$ denote the average sum-rate of the network, we have

$$
\bar{R}_{\text {sum }}=\sum_{j=1}^{M} \bar{R}_{\text {sum }}^{(j)}
$$

where $\bar{R}_{\text {sum }}^{(j)}$ is the average sum-rate of cluster $\mathcal{C}_{j}$ and is given by

$$
\begin{aligned}
\bar{R}_{\text {sum }}^{(j)} & =\mathbb{E}\left[\sum_{i=1}^{n} R_{i}^{(j)}\right] \\
& =\sum_{i=1}^{n} \mathbb{E}\left[\frac{W}{M} \log \left(1+\frac{h_{i i}^{(j)} p_{i}}{I_{i}^{(j)}+\frac{N_{0} W}{M}}\right)\right],
\end{aligned}
$$


where the expectation is computed with respect to $h_{i i}^{(j)}$ and $I_{i}^{(j)}$. We also define the

probability of the link activation in each cluster as $q_{n} \triangleq \operatorname{Pr}\left\{h_{i i}^{(j)}>\tau_{n}\right\}$ that is a function of $n$. Following the same approach as in [1] and [7] with $M=1$ and $\alpha=1$, we can easily prove the following lemmas.

Lemma 1: Assuming $0<\alpha \leq 1$ is fixed, with probability one (w. p. 1), we have

$$
I_{i}^{(j)} \sim \alpha(n-1) q_{n}
$$

as $n \rightarrow \infty$.

Proof: See Appendix I.

Lemma 2: Let $M \sim o(K)$ and $0<\alpha \leq 1$ is a fixed parameter. Then for large values of $K$, the optimum threshold level that maximizes the average sum-rate of each cluster is obtained as

$$
\tau_{n}^{*}=\log \alpha n-2 \log \log \alpha n+O(1) .
$$

Proof: See Appendix II.

Lemma 3: Under the assumptions in Lemma 2, the probability of the link activation in each cluster is given by

$$
q_{n}=c \frac{(\log \alpha n)^{2}}{\alpha n}
$$

where $c$ is a constant.

Proof: Under a Rayleigh fading channel condition, we have

$$
q_{n}=\operatorname{Pr}\left\{h_{i i}^{(j)}>\tau_{n}\right\}=e^{-\tau_{n}} .
$$

Using (4), it is concluded

$$
q_{n}=\frac{(\log \alpha n)^{2}}{\alpha n} \times e^{-O(1)} .
$$

Setting $c=e^{-O(1)}$, we obtain (5).

Lemma 4: Assuming $M \sim o(K)$ and $0<\alpha \leq 1$ is fixed, the maximum achievable throughput of the network is given by

$$
\begin{aligned}
\bar{R}_{\text {sum }} & \approx \frac{W}{\alpha}\left(-\log q_{n}+O(1)\right) \\
& =\frac{W}{\alpha}\left(\log \frac{K}{M}+o(\log K)+O(1)\right) .
\end{aligned}
$$


Proof: See Appendix III.

Lemma 4 states that the average sum-rate of the network for $M \sim o(K)$ depends on the value of $0<\alpha \leq 1$ and scales as $\frac{W}{\alpha} \log \frac{K}{M}$.

Corollary 1: For values of $M$ such that $\log M \sim o(\log K)$, the average sum-rate of the network scales as $\frac{W}{\alpha} \log K$.

\section{Network Analysis with On-Off Power Allocation Strategy}

In this section, we analyze the average sum-rate of the network in terms of $M$, $1 \leq M \leq K$. We prove that the maximum throughput of the network for every value of $0<\alpha \leq 1$ is obtained at $M=1$.

Theorem 1: Assuming $0<\alpha \leq 1$ is fixed, the maximum average sum-rate of the network is achieved at $M=1$.

Proof: We prove the theorem in the following two cases:

Case 1: $M \sim o(K)$

From (6), the average sum-rate of the network for $M \sim o(K)$ is obtained as

$$
\bar{R}_{\text {sum }} \approx \frac{W}{\alpha}\left(-\log q_{n}+O(1)\right) .
$$

Taking the first-order derivative of (8) with respect to $M$ yields,

$$
\frac{\partial \bar{R}_{\text {sum }}}{\partial M}=-\frac{W}{\alpha} \frac{\partial q_{n}}{\partial M} \frac{1}{q_{n}} .
$$

Noting that $n=\frac{K}{M}$, the probability of the link activation is obtained in terms of $M$ by using (5), i.e.,

$$
q_{n}=\frac{c}{\alpha K} M\left(\log \frac{\alpha K}{M}\right)^{2}
$$

Since,

$$
\frac{\partial q_{n}}{\partial M}=\frac{c}{\alpha K} \log \frac{\alpha K}{M} \times\left(\log \frac{\alpha K}{M}-2\right)>0,
$$

it is concluded that (8) is a monotically decreasing function of $M$. Thus, the maximum throughput of the network for $M \sim o(K)$ is obtained at $M=1$.

Case 2: $M \sim \Theta(K)$ 
Letting $m_{j}$ denote the number of active links in cluster $\mathcal{C}_{j}$, it is concluded that $m_{j} \in[1, n]$ does not grow with $K$. In this case, the average sum-rate of the network is given by

$$
\bar{R}_{\text {sum }}=\sum_{j=1}^{M} \sum_{i=1}^{m_{j}} \frac{W}{M} \mathbb{E}\left[\log \left(1+\frac{h_{i i}^{(j)}}{I_{i}^{(j)}+\frac{N_{0} W}{M}}\right)\right] .
$$

We first obtain an upper bound for the average sum-rate of the network when $m_{j}=1,1 \leq j \leq M$. Clearly, since only one user in each cluster activates its transmitter, $I_{i}^{(j)}=0$. Hence, the maximum achievable throughput of cluster $\mathcal{C}_{j}$ is obtained as

$$
\bar{R}_{\text {sum }}^{(j)}=\frac{W}{M} \mathbb{E}\left[\log \left(1+\frac{M}{N_{0} W} h_{\max }^{(j)}\right)\right]
$$

where $h_{\text {max }}^{(j)}=\max _{i=1, \ldots, n} h_{i i}^{(j)}$ is a random variable. Since $\log x$ is a concave function of $x$, an upper bound of (11) is obtained through Jensen's inequality, $\mathbb{E}[\log x] \leq$ $\log (\mathbb{E}[x]), x>0$. Thus,

$$
\bar{R}_{\text {sum }}^{(j)} \leq \frac{W}{M} \log \left(1+\frac{M}{N_{0} W} \mathbb{E}[Y]\right),
$$

where $Y \triangleq h_{\max }^{(j)}$. Noting that $h_{i i}^{(j)}$ 's are i.i.d. over $i \in \mathcal{N}$, we have

$$
\begin{aligned}
F_{Y}(y) & =\operatorname{Pr}\{Y \leq y\}, \quad y>0 \\
& =\prod_{i=1}^{n} \operatorname{Pr}\left\{h_{i i}^{(j)} \leq y\right\} \\
& =\left(1-e^{-y}\right)^{n},
\end{aligned}
$$

where $F_{Y}($.$) is the cumulative distribution function (cdf) of Y$. Hence,

$$
\mathbb{E}[Y]=\int_{0}^{\infty} n y e^{-y}\left(1-e^{-y}\right)^{n-1} d y .
$$

Since $\left(1-e^{-y}\right)^{n-1} \leq 1$, we arrive at the following inequality

$$
\mathbb{E}[Y] \leq \int_{0}^{\infty} n y e^{-y} d y=n
$$

Consequently, the upper bound of $\bar{R}_{\text {sum }}^{(j)}$ obtained in (12) can be simplified as

$$
\bar{R}_{\text {sum }}^{(j)} \leq \frac{W}{M} \log \left(1+\frac{K}{N_{0} W}\right) .
$$


For $m_{j}>1$ and due to the shadowing effect with probability $\alpha$, the average sum-rate of cluster $\mathcal{C}_{j}$ can be written as

$$
\bar{R}_{\text {sum }}^{(j)}=\sum_{i=1}^{m_{j}} \frac{W}{M} \mathbb{E}\left[\log \left(1+\frac{h_{i i}^{(j)}}{\sum_{k \neq i}^{m_{j}} v_{k} h_{k i}^{(j)}+\frac{N_{0} W}{M}}\right)\right], \quad k \in \mathcal{C}_{j},
$$

where $v_{k}$ 's are binomial random variables with parameters $\left(m_{j}, \alpha\right)$. Thus,

$$
\begin{aligned}
\bar{R}_{\text {sum }}^{(j)} & =\frac{W}{M} \sum_{i=1}^{m_{j}} \sum_{l=0}^{m_{j}}\left(\begin{array}{c}
m_{j} \\
l
\end{array}\right) \alpha^{l}(1-\alpha)^{m_{j}-l} \mathbb{E}\left[\log \left(1+\frac{h_{i i}^{(j)}}{\Upsilon_{l}+\frac{N_{0} W}{M}}\right)\right] \\
& =\frac{W}{M} \sum_{i=1}^{m_{j}}(1-\alpha)^{m_{j}} \mathbb{E}\left[\log \left(1+\frac{h_{i i}^{(j)}}{\frac{N_{0} W}{M}}\right)\right] \\
& +\frac{W}{M} \sum_{i=1}^{m_{j}} \sum_{l=1}^{m_{j}}\left(\begin{array}{c}
m_{j} \\
l
\end{array}\right) \alpha^{l}(1-\alpha)^{m_{j}-l} \mathbb{E}\left[\log \left(1+\frac{h_{i i}^{(j)}}{\Upsilon_{l}+\frac{N_{0} W}{M}}\right)\right],
\end{aligned}
$$

where $\Upsilon_{l}$ is the sum of $l$ i.i.d random variables with $\chi^{2}(2)$ distribution. Noting that $M \sim \Theta(K)$, we ignore the term $\frac{N_{0} W}{M}$. Also for $m_{j}>1, \Upsilon_{l}$ is greater than the interference term caused by one interfering link. Thus, an upper bound for the throughput of cluster $j$ is given by

$$
\begin{aligned}
\bar{R}_{\text {sum }}^{(j)} & \leq \frac{W}{M} m_{j}(1-\alpha)^{m_{j}} \mathbb{E}\left[\log \left(1+\frac{Y}{\frac{N_{0} W}{M}}\right)\right] \\
& +\frac{W}{M} \sum_{i=1}^{m_{j}} \sum_{l=1}^{m_{j}}\left(\begin{array}{c}
m_{j} \\
l
\end{array}\right) \alpha^{l}(1-\alpha)^{m_{j}-l} \mathbb{E}\left[\log \left(1+\frac{Y}{Z}\right)\right],
\end{aligned}
$$

where $Y \triangleq h_{\max }^{(j)}=\max _{i=1, \ldots, n} h_{i i}^{(j)}$ and $Z \triangleq h_{k i}^{(j)}$. According to binomial formula, we have

$$
\sum_{l=1}^{m_{j}}\left(\begin{array}{c}
m_{j} \\
l
\end{array}\right) \alpha^{l}(1-\alpha)^{m_{j}-l}=1-(1-\alpha)^{m_{j}}
$$

Thus,

$$
\begin{aligned}
\bar{R}_{\text {sum }}^{(j)} & \leq \frac{W}{M} m_{j}(1-\alpha)^{m_{j}} \mathbb{E}\left[\log \left(1+\frac{Y}{\frac{N_{0} W}{M}}\right)\right] \\
& +\frac{W}{M} m_{j}\left(1-(1-\alpha)^{m_{j}}\right) \mathbb{E}\left[\log \left(1+\frac{Y}{Z}\right)\right]
\end{aligned}
$$


Letting $X=\frac{Y}{Z}$, the cdf of $X$ is obtained as

$$
\begin{aligned}
F_{X}(x) & =\operatorname{Pr}\{X \leq x\}, \quad x>0 \\
& =\operatorname{Pr}\{Y \leq Z x\} \\
& =\int_{0}^{\infty} \operatorname{Pr}\{Y \leq Z x \mid Z\} f_{Z}(z) d z \\
& =\int_{0}^{\infty}\left(1-e^{-z x}\right)^{n} e^{-z} d z .
\end{aligned}
$$

Thus, the probability distribution function (pdf) of $X$ can be written as

$$
\begin{aligned}
f_{X}(x) & =\frac{d F_{X}(x)}{d x} \\
& =\int_{0}^{\infty} n z e^{-z(1+x)}\left(1-e^{-z x}\right)^{n-1} d z \\
& \leq \int_{0}^{\infty} n z e^{-z(1+x)} d z=\frac{n}{(1+x)^{2}} .
\end{aligned}
$$

Using (12)-(14) and (19), the inequality (18) is simplified as

$$
\begin{aligned}
\bar{R}_{\text {sum }}^{(j)} & \leq \frac{W}{M} m_{j}(1-\alpha)^{m_{j}} \log \left(1+\frac{K}{N_{0} W}\right) \\
& +\frac{W}{M} m_{j}\left(1-(1-\alpha)^{m_{j}}\right) \int_{0}^{\infty} \log (1+x) f_{X}(x) d x \\
& \leq \frac{W}{M} m_{j}(1-\alpha)^{m_{j}} \log \left(1+\frac{K}{N_{0} W}\right) \\
& +\frac{W}{M} n m_{j}\left(1-(1-\alpha)^{m_{j}}\right) \int_{0}^{\infty} \frac{\log (1+x)}{(1+x)^{2}} d x \\
& =\frac{W}{M} m_{j}(1-\alpha)^{m_{j}} \log \left(1+\frac{K}{N_{0} W}\right)+\Lambda .
\end{aligned}
$$

where $\Lambda=\frac{W}{M} n m_{j}\left(1-(1-\alpha)^{m_{j}}\right)$ and it does not grows with $K$. Let's assume that among $M$ clusters, $\Gamma$ clusters have $m_{j}=1$ and for the $M-\Gamma$ of the rest, the number of active links in each cluster is greater than one. Hence by using (14) and (20), an upper bound for the network throughput is obtained as

$$
\begin{aligned}
\bar{R}_{\text {sum }} & \leq \Gamma \frac{W}{M} \log \left(1+\frac{K}{N_{0} W}\right)+(M-\Gamma) \frac{W}{M} m_{j}(1-\alpha)^{m_{j}} \log \left(1+\frac{K}{N_{0} W}\right) \\
& +(M-\Gamma) \frac{W}{M} n m_{j}\left(1-(1-\alpha)^{m_{j}}\right) .
\end{aligned}
$$


Since $0<\alpha \leq 1$, it is clear that

$$
\frac{\Gamma W}{M} \log \left(1+\frac{K}{N_{0} W}\right) \leq \frac{\Gamma W}{M \alpha} \log \left(1+\frac{K}{N_{0} W}\right) .
$$

In order to show that the achievable average sum-rate obtained in (21) is less than that of $M=1$ in (7), it is sufficient to prove

$$
(M-\Gamma) \frac{W}{M} m_{j}(1-\alpha)^{m_{j}} \log \left(1+\frac{K}{N_{0} W}\right)<(M-\Gamma) \frac{W}{M \alpha} \log \left(1+\frac{K}{N_{0} W}\right),
$$

or

$$
m_{j}(1-\alpha)^{m_{j}}<\frac{1}{\alpha}
$$

Letting $f(\alpha)=\alpha m_{j}(1-\alpha)^{m_{j}}$, we have

$$
\frac{\partial f(\alpha)}{\partial \alpha}=m_{j}(1-\alpha)^{m_{j}-1}\left(1-\alpha-\alpha m_{j}\right)=0 .
$$

Thus, the extremum points of $f(\alpha)$ are located at $\alpha=1$ and $\alpha=\frac{1}{m_{j}+1}$. It is seen that

$$
f(1)=0<1
$$

and

$$
f\left(\frac{1}{m_{j}+1}\right)=\left(\frac{m_{j}}{m_{j}+1}\right)^{m_{j}+1}<1 .
$$

Since $f(\alpha)<1$, we can conclude that the network throughput for $M \sim \Theta(K)$ is less than that of $M=1$.

Corollary 2: Although in the proof of Theorem 1, it is assumed that $\alpha$ is fixed, in the case of $M \sim \Theta(K)$ and following the same proof steps, it can be shown that for $\alpha \rightarrow 0$ the throughput of the network is less than that of $M=1$.

Note that for $M \sim \Theta(K)$, which includes $M=K$, we obtained an upper bound for $\bar{R}_{\text {sum }}$. In the next corollary, we derive the exact achievable throughput of the network for $M=K$.

Corollary 3: Assuming $M=K$, the average sum-rate of the network is obtained by

$$
\bar{R}_{\text {sum }} \approx W\left(\log K-\log N_{0} W-\gamma\right)
$$


where $\gamma$ is Euler's constant.

Proof: Noting that for $M=K$, only one user exists in each cluster, all the users can transmit with full power over the orthogonal subchannels. Hence, since $I_{i}^{(j)}=0$, for $i=1, \ldots, K$, the average sum-rate of the network is given by

$$
\begin{aligned}
\bar{R}_{\text {sum }} & =\mathbb{E}\left[\sum_{i=1}^{K} R_{i}^{(j)}\right] \\
& =\frac{W}{K} \sum_{i=1}^{K} \mathbb{E}\left[\log \left(1+\frac{h_{i i}^{(j)}}{\frac{N_{0} W}{K}}\right)\right],
\end{aligned}
$$

where the expectation is computed with respect to $h_{i i}^{(j)}$. Under a Rayleigh fading channel model, we have

$$
\bar{R}_{\text {sum }}=\frac{W}{K} \sum_{i=1}^{K} \int_{0}^{\infty} e^{-x} \log (1+\lambda x) d x,
$$

where $\lambda \triangleq \frac{K}{N_{0} W}$. Thus,

$$
\begin{aligned}
\bar{R}_{\text {sum }} & =W \int_{0}^{\infty} e^{-x} \log (1+\lambda x) d x \\
& =W e^{\frac{1}{\lambda}} \int_{1 / \lambda}^{\infty} \frac{e^{-x}}{x} d x \\
& =W e^{\frac{1}{\lambda}} \mathrm{E}_{1}\left(\frac{1}{\lambda}\right)
\end{aligned}
$$

where $\mathrm{E}_{1}(x)$ is obtained by the exponential-integral function defined as [14]

$$
\mathrm{E}_{n}(x) \triangleq \int_{1}^{\infty} \frac{e^{-t x}}{t^{n}} d t
$$

To simplify (22), we use the following series representation for $\mathrm{E}_{1}(x)$,

$$
\mathrm{E}_{1}(x)=-\gamma-\log x+\sum_{s=1}^{\infty} \frac{(-1)^{s+1} x^{s}}{s . s !},
$$

where $\gamma$ is Euler's constant and is defined by the limit [14]

$$
\gamma=\lim _{s \rightarrow \infty}\left(\sum_{k=1}^{s} \frac{1}{k}-\log s\right)=0.577215665 \ldots
$$


Thus, the average sum-rate of the network is obtained as

$$
\bar{R}_{\text {sum }}=W e^{\frac{1}{\lambda}}\left(-\gamma+\log \lambda+\sum_{s=1}^{\infty} \frac{(-1)^{s+1}}{s . s !}\left(\frac{1}{\lambda}\right)^{s}\right) .
$$

For sufficiently large values of $K$, we have $\lambda=\frac{K}{N_{0} W} \gg 1$, which results in $e^{\frac{1}{\lambda}} \approx 1$ and

$$
\sum_{s=1}^{\infty} \frac{(-1)^{s+1}}{s . s !}\left(\frac{1}{\lambda}\right)^{s} \approx 0
$$

Consequently for $M=K$, the average sum-rate of the network is asymptotically obtained by

$$
\bar{R}_{\text {sum }} \approx W\left(\log K-\log N_{0} W-\gamma\right)
$$

Corollary 4: For $M=K$, the average sum-rate of the network is of order $\log K$. Through comparing (24) with (7), it is concluded that the throughput of the network with $M=K$ is less than or equal to that of $M=1$.

We finally evaluate the throughput of the network versus the number of clusters for finite values of $K$ through simulation results. Fig. 1 illustrates the maximum average sum-rate of the network versus $M$ for $K=20, K=40$ and $\alpha=0.1$. From the figure, we can see that the average sum-rate is a decreasing function of $M$. So, the maximum value of $\bar{R}_{\text {sum }}$ is achieved at $M=1$.

\section{CONCLUSion}

We have analyzed the average sum-rate of a single-hop wireless network in terms of the number of clusters, $M$, and under the shadowing effect with probability $\alpha$. It has been demonstrated that for $M \sim o(K)$ and $0<\alpha \leq 1$, where $\alpha$ is fixed, the average sum-rate of the network is of order $\frac{W}{\alpha} \log \frac{K}{M}$. Also, it has been proved that the maximum average sum-rate of the network for every value of $0<\alpha \leq 1$ and $1 \leq M \leq K$ is achieved at $M=1$. In fact, in the proposed model, partitioning the bandwidth $W$ into $M$ subchannels has no gain in terms of enhancing the throughput. 


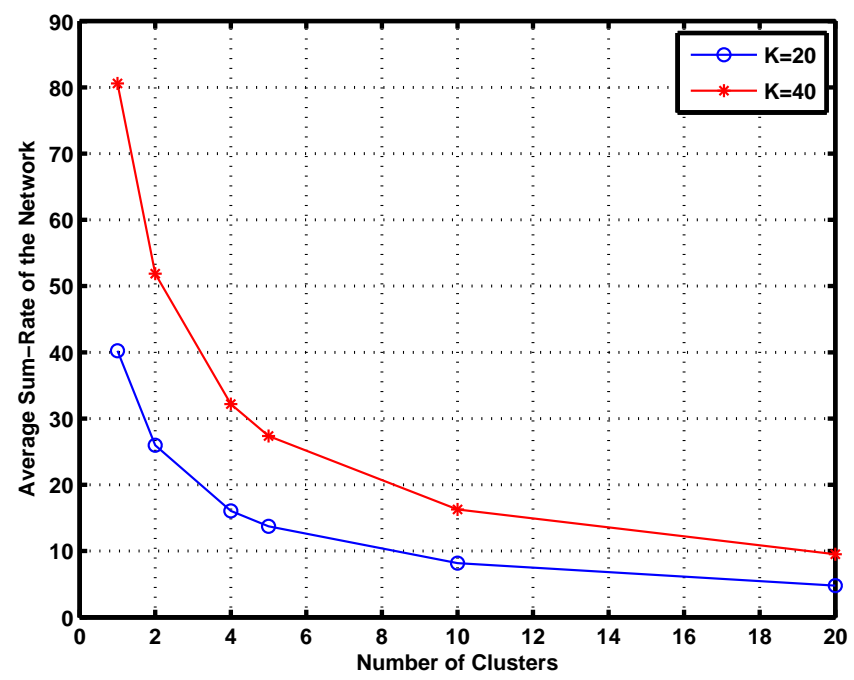

Fig. 1. Average sum-rate of the network vs. $M$ for $K=20$ and $K=40$.

Throughout this work, it is assumed that all the links use a single antenna. A possible future extension of this work would be to analyze the performance of the network with multiple antenna transmitters/receivers. Also, we showed that the maximum throughput is achieved at $M=1$ without considering the fairness issues. An interesting problem for future research is to analyze the tradeoff between the maximum throughput of the network and the fairness between the users in terms of $M$.

\section{APPENDIX I}

\section{Proof of LEMMA 1}

Let define $\chi_{k} \triangleq \mathcal{L}_{k i}^{(j)} p_{k}$, where $\mathcal{L}_{k i}^{(j)}$ is independent of $p_{k}$, for $k \neq i$. Under a quasi-static Rayleigh fading channel condition, it is concluded that $\chi_{k}$ 's are the i.i.d. random variables with

$$
\begin{aligned}
\mathbb{E}\left[\chi_{k}\right] & =\mathbb{E}\left[\mathcal{L}_{k i}^{(j)} p_{k}\right]=\alpha q_{n}, \\
\operatorname{Var}\left[\chi_{k}\right] & =\mathbb{E}\left[\chi_{k}^{2}\right]-\mathbb{E}^{2}\left[\chi_{k}\right]=2 \alpha q_{n}-\left(\alpha q_{n}\right)^{2},
\end{aligned}
$$


where $\mathbb{E}\left[\left(h_{k i}^{(j)}\right)^{2}\right]=2$ and $\mathbb{E}\left[p_{k}\right]=q_{n}$. Also, the interference $I_{i}^{(j)}$ is a random variable with mean $\mu_{n}$ and variance $\vartheta_{n}^{2}$, where

$$
\begin{aligned}
\mu_{n} & =\mathbb{E}\left[\sum_{\substack{k \neq i \\
k=1}}^{n} \chi_{k}\right]=\alpha(n-1) q_{n}, \\
\vartheta_{n}^{2} & =(n-1)\left(2 \alpha q_{n}-\left(\alpha q_{n}\right)^{2}\right) .
\end{aligned}
$$

By using the Chebyshev inequality, we obtain

$$
\operatorname{Pr}\left\{\left|I_{i}^{(j)}-\mu_{n}\right|<\psi_{n}\right\} \geq 1-\frac{\vartheta_{n}^{2}}{\psi_{n}^{2}}
$$

for all $\psi_{n}>0$. Thus, we have

$$
\operatorname{Pr}\left\{\left|I_{i}^{(j)}-\alpha(n-1) q_{n}\right|<\psi_{n}\right\} \geq 1-\frac{(n-1)\left(2 \alpha q_{n}-\left(\alpha q_{n}\right)^{2}\right)}{\psi_{n}^{2}} .
$$

It is seen that for

$$
\psi_{n}=\omega\left(\sqrt{(n-1)\left(2 \alpha q_{n}-\left(\alpha q_{n}\right)^{2}\right)}\right)
$$

we have

$$
\lim _{n \rightarrow \infty} 1-\frac{(n-1)\left(2 \alpha q_{n}-\left(\alpha q_{n}\right)^{2}\right)}{\psi_{n}^{2}}=1
$$

Thus,

$$
\alpha(n-1) q_{n}-\psi_{n}<I_{i}^{(j)}<\alpha(n-1) q_{n}+\psi_{n}, \quad \text { w.p. } 1 .
$$

By choosing $\psi_{n}=o\left(\alpha(n-1) q_{n}\right)$, we can obtain $I_{i}^{(j)} \sim \alpha(n-1) q_{n}$, w. p. 1 .

\section{Appendix II}

\section{Proof of Lemma 2}

Under the on-off power allocation strategy and using $q_{n}=\operatorname{Pr}\left\{h_{i i}^{(j)}>\tau_{n}\right\}$, we have

$$
\begin{aligned}
\mathbb{E}\left[R_{i}^{(j)}\right] & =\mathbb{E}\left[R_{i}^{(j)} \mid h_{i i}^{(j)}>\tau_{n}\right] \operatorname{Pr}\left\{h_{i i}^{(j)}>\tau_{n}\right\}+\mathbb{E}\left[R_{i}^{(j)} \mid h_{i i}^{(j)} \leq \tau_{n}\right] \operatorname{Pr}\left\{h_{i i}^{(j)} \leq \tau_{n}\right\} \\
& =q_{n} \mathbb{E}\left[R_{i}^{(j)} \mid h_{i i}^{(j)}>\tau_{n}\right]+\left(1-q_{n}\right) \mathbb{E}\left[R_{i}^{(j)} \mid h_{i i}^{(j)} \leq \tau_{n}\right] .
\end{aligned}
$$


Since for $h_{i i}^{(j)} \leq \tau_{n}, p_{i}=0$, it is concluded

$$
\mathbb{E}\left[R_{i}^{(j)}\right]=\frac{q_{n} W}{M} \mathbb{E}\left[\log \left(1+\frac{h_{i i}^{(j)}}{I_{i}^{(j)}+\frac{N_{0} W}{M}}\right) \mid h_{i i}^{(j)}>\tau_{n}\right] .
$$

Under condition $M \sim o(K)$ and for large values of $K$, the number of links in each cluster is sufficiently large. So, we can apply Lemma 1 to obtain

$$
\mathbb{E}\left[R_{i}^{(j)}\right] \approx \frac{q_{n} W}{M} \mathbb{E}\left[\log \left(1+\frac{h_{i i}^{(j)}}{\alpha(n-1) q_{n}+\frac{N_{0} W}{M}}\right) \mid h_{i i}^{(j)}>\tau_{n}\right],
$$

where the expectation is computed with respect to $h_{i i}^{(j)}$. For large values of $n$, we can ignore the noise power $\frac{N_{0} W}{M}$. Hence, by using the approximation $\log (1+z) \approx z-\frac{z^{2}}{2}$ for $|z| \ll 1$, we have

$$
\mathbb{E}\left[R_{i}^{(j)}\right] \approx \frac{q_{n} W}{M}\left\{\frac{1}{\alpha n q_{n}} \mathbb{E}\left[h_{i i}^{(j)} \mid h_{i i}^{(j)}>\tau_{n}\right]-\frac{1}{2} \frac{1}{\left(\alpha n q_{n}\right)^{2}} \mathbb{E}\left[\left(h_{i i}^{(j)}\right)^{2} \mid h_{i i}^{(j)}>\tau_{n}\right]\right\} .
$$

Under a Rayleigh fading channel model,

$$
\begin{gathered}
\mathbb{E}\left[h_{i i}^{(j)} \mid h_{i i}^{(j)}>\tau_{n}\right]=1+\tau_{n}, \\
\mathbb{E}\left[\left(h_{i i}^{(j)}\right)^{2} \mid h_{i i}^{(j)}>\tau_{n}\right]=\tau_{n}^{2}+2 \tau_{n}+2 .
\end{gathered}
$$

Using $q_{n}=\operatorname{Pr}\left\{h_{i i}^{(j)}>\tau_{n}\right\}=e^{-\tau_{n}}$, the average sum-rate of the links in $\mathcal{C}_{j}$ is given by

$$
\begin{aligned}
\bar{R}_{\text {sum }}^{(j)} & =\sum_{i=1}^{n} \mathbb{E}\left[R_{i}^{(j)}\right] \\
& =\frac{W}{\alpha M}\left[1+\tau_{n}-\frac{\tau_{n}^{2}+2 \tau_{n}+2}{2 \alpha n e^{-\tau_{n}}}\right] .
\end{aligned}
$$

Thus, the optimization problem is

$$
\tau_{n}^{*}=\arg \max _{\tau_{n}} \bar{R}_{\text {sum }}^{(j)}
$$

Taking the first-order derivative of (B-3) yields

$$
\frac{\partial \bar{R}_{s u m}^{(j)}}{\partial \tau_{n}}=\frac{W}{\alpha M}\left[1-\frac{\tau_{n}^{2}+4 \tau_{n}+4}{2 \alpha n e^{-\tau_{n}}}\right] .
$$


Since, the second-order derivative of (B-3) is negative, the maximum value of $\bar{R}_{\text {sum }}^{(j)}$ is obtained by setting (B-4) equal to zero. So, we have

$$
2 \alpha n e^{-\tau_{n}}=\tau_{n}^{2}+4 \tau_{n}+4
$$

or

$$
\tau_{n}=\log 2 \alpha n-2 \log \tau_{n}-\log \left(1+\frac{4 \tau_{n}+4}{\tau_{n}^{2}}\right) .
$$

It can be verified that the solution for (B-5) is

$$
\tau_{n}^{*}=\log \alpha n-2 \log \log \alpha n+O(1)
$$

\section{Appendix III}

\section{Proof of Lemma 4}

Using (B-3), the network throughput is obtained as

$$
\begin{aligned}
\bar{R}_{\text {sum }} & =\sum_{j=1}^{M} \bar{R}_{\text {sum }}^{(j)} \\
& =\frac{W}{\alpha}\left[1+\tau_{n}-\frac{\tau_{n}^{2}+2 \tau_{n}+2}{2 \alpha n e^{-\tau_{n}}}\right] .
\end{aligned}
$$

Considering the optimum threshold level obtained in (B-5) and noting that $\alpha n e^{-\tau_{n}}=\alpha n q_{n}=c(\log \alpha n)^{2}$, it can be easily shown that for $M \sim o(K)$

$$
1-\frac{\tau_{n}^{2}+2 \tau_{n}+2}{2 \alpha n e^{-\tau_{n}}} \sim O(1) .
$$

Hence by using $\tau_{n}=-\log q_{n}$, we arrive at the following equation

$$
\bar{R}_{\text {sum }} \approx \frac{W}{\alpha}\left[-\log q_{n}+O(1)\right]
$$

Through substituting (5) in (C-2) and using $n=\frac{K}{M}$, we finally obtain

$$
\bar{R}_{\text {sum }}=\frac{W}{\alpha}\left(\log \frac{K}{M}+o(\log K)+O(1)\right) .
$$




\section{REFERENCES}

[1] M. Ebrahimi, M. A. Maddah-Ali, and A. K. Khandani, "Power allocation and asymptotic achievable sum-rates in single-hop wireless networks," in Proc. 40th Conference on Information Sciences and Systems (CISS'06), Princeton University, Princton, NJ, March 2006.

[2] A. Sampath, P. Sarath Kumar, and J. M. Holtzman, "Power control and resource management for a multimedia CDMA wireless system," in Proc. IEEE PIMRC'95, Sept. 1995, vol. 1, pp. 21-25.

[3] I. Katzela and M. Naghshineh, "Channel assignment schemes for cellular mobile telecommunication systems: a comprehensive survey," IEEE Personal Communications, vol. 3, no. 3, pp. 10-31, June 1996.

[4] O. Seong-Jun, D. Zhang, and K. M. Wasserman, "Optimal resource allocation in multiservice CDMA networks," IEEE Trans. on Wireless Commun., vol. 2, no. 4, pp. 811-821, July 2003.

[5] Z. Han, Z. Ji, and K. J. R. Liu, "Fair multiuser channel allocation for OFDMA networks using nash bargaining solutions and coalitions," IEEE Trans. on Communications, vol. 53, no. 8, pp. 1366-1376, August 2005.

[6] J. Huang, R. A. Berry, and M. L. Honig, "Distributed interference compensation for wireless networks," IEEE Journal on Selected Areas in Communications, vol. 24, no. 5, pp. 1074 - 1084, May 2006.

[7] J. Abouei, M. Ebrahimi, and A. K. Khandani, "A new decentralized power allocation strategy in singlehop wireless networks," in Proc. IEEE 41st Conference on Information Sciences and Systems (CISS'07), Johns Hopkins University, Baltimore, MD, USA, March 2007.

[8] R. Etkin, A. Parekh, and D. Tse, "Spectrum sharing in unlicensed bands," in IEEE Dyspan, Baltimore MD, 2005.

[9] R. Gowaikar, B. Hochwald, and B. Hassibi, "Communication over a wireless network with random connections," IEEE Trans. on Information Theory, vol. 52, no. 7, pp. 2857-2871, July 2006.

[10] S. G. Kiani and D. Gesbert, "Maximizing the capacity of large wireless networks: optimal and distributed solutions," in Proc. IEEE International Symposium on Information Theory (ISIT), Seattle, USA, July 2006, pp. $2501-2505$.

[11] A. Gjendemsj, D. Gesbert, G. E. $\phi$ ien, and S. G. Kiani, "Optimal power allocation and scheduling for two-cell capacity maximization," in Proc. IEEE Symposium on Modeling and Optimization in Mobile, Ad-Hoc and Wireless Networks, Boston, MA, April 2006.

[12] C. Bettstetter and C. Hartmann, "Connectivity of wireless multihop networks in a shadow fading environment," ACM/Kluwer Wireless Networks, Special Issue on Modeling and Analysis of Mobile Networks, vol. 11, no. 4, July 2005.

[13] D. E. Knuth, "Big omicron and big omega and big theta," in ACM SIGACT News, April-June 1967, vol. 8 , pp. $18-24$.

[14] I. S. Gradshteyn, I. M. Ryzhik, and A. Jeffrey, Table of Integrals, Series, and Products, Academic Press, 1994. 